\title{
Knowledge-based Design of 5-Flouroracil Prodrug Liposomal Formulation: Molecular Packing and Interaction Revealed by Interfacial Isotherms and X-ray Scattering Techniques
}

Tiep Pham ${ }^{1}$, Paola Leon Plata ${ }^{1}$, Pin Zhang ${ }^{1}$, Anand Vellara ${ }^{1}$, Wei Bu², Binhua Lin ${ }^{2}$, Gang Cheng ${ }^{1}$, and Ying Liu ${ }^{1,3,4, *}$

${ }^{1}$ Department of Chemical Engineering, University of Illinois at Chicago, Chicago, Illinois 60608, USA;

${ }^{2}$ NSF's ChemMatCARS, University of Chicago, Chicago, Illinois 60637, USA;

${ }^{3}$ Department of Bioengineering, University of Illinois at Chicago, Chicago, Illinois 60607, USA

${ }^{4}$ Department of Biopharmaceutical Sciences, University of Illinois at Chicago, Chicago, Illinois 60607, USA

*Corresponding should be sent to: $\underline{\text { liuying@uic.edu }}$

Table S1. Virial coefficients from fitting the curves shown in Figure 2C, for the mixtures of DSTAP and 5-FCPal at various ratios.

\begin{tabular}{lcccccc}
\multicolumn{1}{c}{$\chi_{5-\mathrm{FCPa}}$} & \multicolumn{5}{c}{} \\
\cline { 2 - 7 } & 0 & 0.3 & 0.5 & 0.67 & 0.77 & 1 \\
\hline$b_{0}$ & 0.0181 & 0.0657 & 0.1906 & 0.0794 & 0.0934 & 0.3091 \\
$b_{1}$ & 0.1218 & 0.1364 & 0.1498 & 0.1570 & 0.1685 & 0.1663 \\
$b_{2}$ & -0.0004 & -0.0010 & -0.0015 & -0.0028 & -0.0035 & -0.0039 \\
$R^{2}$ & 0.9997 & 0.9979 & 0.9925 & 0.9919 & 0.9892 & 0.9991
\end{tabular}

Table S2. Virial coefficients from fitting the curves shown in Figure 5C, for the mixtures of DOTAP and 5-FCPal at various ratios.

\begin{tabular}{lcccccc}
\multicolumn{1}{c}{$\chi_{5 \text {-FCPal }}$} & \multicolumn{5}{c}{} \\
\cline { 2 - 7 } & 0 & 0.3 & 0.5 & 0.67 & 0.77 & 1 \\
\hline$b_{0}$ & 0.1421 & 0.1369 & 0.1740 & 0.1346 & 0.1358 & 0.3091 \\
$b_{1}$ & 0.227 & 0.2125 & 0.2004 & 0.1759 & 0.1608 & 0.1663 \\
$b_{2}$ & -0.0023 & -0.0027 & -0.0027 & -0.0028 & -0.0027 & -0.0039 \\
$R^{2}$ & 0.9998 & 0.9992 & 0.9984 & 0.9951 & 0.9917 & 0.9991
\end{tabular}


Table S3. Virial coefficients from fitting the curves shown in Figure 7C, for the mixtures of DPPC and 5-FCPal at various ratios.

\begin{tabular}{cccccc}
\multicolumn{7}{c}{$\chi_{\text {5-FCPal }}$} & \multicolumn{5}{l}{} \\
\cline { 2 - 6 } & 0 & 0.3 & 0.5 & 0.67 & 1 \\
\hline$b_{0}$ & 0.1124 & 0.1262 & 0.1267 & 0.0765 & 0.3091 \\
$b_{1}$ & 0.1302 & 0.1605 & 0.1570 & 0.1602 & 0.1663 \\
$b_{2}$ & -0.0008 & -0.0018 & -0.0021 & -0.0025 & -0.0039 \\
$R^{2}$ & 0.9938 & 0.9940 & 0.9846 & 0.99091 & 0.9991
\end{tabular}

Table S4. Virial coefficients from fitting the curves shown in Figure 9C, for the mixtures of DPPG and 5-FCPal at various ratios.

\begin{tabular}{cccccc}
\multicolumn{7}{c}{$\chi_{5-\mathrm{FCPal}}$} & \multicolumn{5}{c}{} \\
\cline { 2 - 6 } & 0 & 0.3 & 0.5 & 0.67 & 1 \\
\hline$b_{0}$ & 0.8181 & 0.4038 & 0.3596 & 0.2101 & 0.3091 \\
$b_{1}$ & 0.1216 & 0.1714 & 0.1749 & 0.1787 & 0.1663 \\
$b_{2}$ & -0.0007 & -0.0020 & -0.0023 & -0.0025 & -0.0039 \\
$R^{2}$ & 0.9635 & 0.9782 & 0.9791 & 0.9821 & 0.9991
\end{tabular}

Table S5. GIXD fitting parameters for DSTAP and the mixtures of DSTAP and 5-FCPal ( $\mathrm{x}_{5-\mathrm{FCPal}}$ $=0.3,0.5,0.67$ and 0.77 ) monolayers at constant pressure of $25 \mathrm{mN} / \mathrm{m}$ at the air - liquid interface.

\begin{tabular}{lccccc} 
& $\mathrm{Q}_{\mathrm{xy}}[1,1]$ & $\mathrm{Q}_{\mathrm{xy}}[0,2]$ & $\mathrm{Q}_{\mathrm{Z}}[1,1]$ & Area $\left(\AA^{2}\right)$ & $\mathrm{t}[$ degrees $]$ \\
\hline $\mathrm{x}_{\text {5-FCPal }}=0$ & $1.37^{ \pm 0.002}$ & $1.46^{ \pm 0.0005}$ & $0.75^{ \pm 0.01}$ & $46.80^{ \pm 0.3}$ & $32.92^{ \pm 0.3}$ \\
$\mathrm{x}_{\text {5-FCPal }}=0.3$ & $1.40^{ \pm 0.001}$ & $1.47^{ \pm 0.001}$ & $0.64^{ \pm 0.004}$ & $44.87^{ \pm 0.3}$ & $28.26^{ \pm 0.1}$ \\
$\mathrm{x}_{\text {5-FCPal }}=0.5$ & $1.42^{ \pm 0.001}$ & $1.48^{ \pm 0.001}$ & $0.57^{ \pm 0.003}$ & $43.4^{ \pm 0.3}$ & $25.08^{ \pm 0.1}$ \\
$\mathrm{x}_{\text {5-FCPal }}=0.67$ & $1.42^{ \pm 0.002}$ & $1.48^{ \pm 0.001}$ & $0.58^{ \pm 0.003}$ & $44.2^{ \pm 0.3}$ & $25.67^{ \pm 0.1}$ \\
$\mathrm{x}_{\text {5-FCPal }}=0.77$ & $1.43^{ \pm 0.001}$ & $1.48^{ \pm 0.001}$ & $0.55^{ \pm 0.003}$ & $43.69^{ \pm 0.4}$ & $24.39^{ \pm 0.1}$
\end{tabular}

Area is the molecular area in the xy-plane, $t$ is the tilt angle of the molecules in degree. 
Table S6. Structural parameters from model fitting of X-ray reflectivity curves for DSTAP and the mixtures of DSTAP and 5-FCPal $\left(\mathrm{x}_{5-\mathrm{FCPal}}=0.3,0.5,0.67\right.$ and 0.77$)$ monolayers at constant pressure of $25 \mathrm{mN} / \mathrm{m}$ at the air - liquid interface.

\begin{tabular}{|c|c|c|c|c|c|}
\hline $\mathbf{x}_{\text {5-FCPal }}$ & $\mathbf{0}$ & 0.3 & 0.5 & 0.67 & 0.77 \\
\hline$\sigma_{0}(\AA)$ & $2.9_{-0.121}^{+0.019}$ & $2.9_{-0.021}^{+0.039}$ & $3.2_{-0.02}^{+0.15}$ & $3.4_{-0.054}^{+0.301}$ & $3.7_{-0.214}^{+0.055}$ \\
\hline $\mathrm{d}_{\text {head } 1}(\AA)$ & $3.7_{-1.789}^{+1.311}$ & $6.8_{-3.7}^{+2.0}$ & $6.3_{-3.275}^{+4.025}$ & $9.1_{-2.426}^{+2.124}$ & $9.2_{-0.628}^{+1.872}$ \\
\hline$\rho_{\text {head } 1}\left(\mathrm{e} \AA^{-3}\right)$ & $0.47_{-0.024}^{+0.106}$ & $0.45_{-0.005}^{+0.036}$ & $0.48_{-0.004}^{+0.005}$ & $0.47_{-0.012}^{+0.109}$ & $0.48_{-0.002}^{+0.004}$ \\
\hline $\mathrm{d}_{\text {head2 } 2}(\AA)$ & $2.9_{-1.412}^{+1.188}$ & $8.7_{-1.263}^{+3.337}$ & $6.2_{-0.379}^{+4.821}$ & $10.2_{-0.66}^{+0.24}$ & $12.4_{-0.37}^{+0.23}$ \\
\hline$\rho_{\text {head2 }}\left(\mathrm{e} \AA^{-3}\right)$ & $0.36_{-0.015}^{+0.046}$ & $0.35_{-0.007}^{+0.002}$ & $0.38_{-0.015}^{+0.007}$ & $0.35_{-0.006}^{+0.009}$ & $0.35_{-0.003}^{+0.002}$ \\
\hline $\mathrm{d}_{\text {tail1 }}(\AA)$ & $9.5_{-0.943}^{+1.457}$ & $17.9_{-0.14}^{+0.46}$ & $18.8_{-0.11}^{+0.89}$ & $18.0_{-0.21}^{+1.99}$ & $18.3_{-0.78}^{+0.51}$ \\
\hline$\rho_{\text {tail1 }}\left(\mathrm{e} \AA^{-3}\right)$ & $0.32_{-0.005}^{+0.005}$ & $0.32_{-0.002}^{+0.003}$ & $0.33_{-0.002}^{+0.003}$ & $0.32_{-0.003}^{+0.009}$ & $0.31_{-0.002}^{+0.003}$ \\
\hline $\mathrm{d}_{\text {tail2 }}(\AA)$ & $8.5_{-0.688}^{+1.562}$ & & & & \\
\hline$\rho_{\text {tail2 }}\left(\mathrm{e} \AA^{-3}\right)$ & $0.32_{-0.003}^{+0.001}$ & & & & \\
\hline
\end{tabular}

The electron density of the aqueous phase was 0.336 e $\AA^{-3}$ for all samples. The error bar for each parameter was calculated based on one standard deviation from the best fit value. The electron density of the alkyl chain tail was constrained to the range $0-0.34$ e $\AA^{-3}$. The thickness of the head group of DSTAP was constrained to 5-6 $\AA$. The thickness of the head group in the subphase for DSTAP-5-FCPal was constrained to not exceed 15-17 $\AA$.

Table S7. Structural fitting parameters for pure prodrug at the air-liquid interface at $10 \mathrm{mN} / \mathrm{m}$, $15 \mathrm{mN} / \mathrm{m}$ and $18.5 \mathrm{mN} / \mathrm{m}$.

\begin{tabular}{llll} 
& $\mathbf{1 0} \mathbf{m N} / \mathbf{m}$ & $\mathbf{1 5} \mathbf{m N} / \mathbf{m}$ & $\mathbf{1 8 . 5} \mathbf{m N} / \mathbf{m}$ \\
\hline$\sigma_{0}(\AA)$ & $3.1_{-0.394}^{+0.456}$ & $3.1_{-0.215}^{+0.105}$ & $3.0_{-0.427}^{+0.073}$ \\
$\mathrm{~d}_{\text {head1 }}(\AA)$ & $4.2_{-0.865}^{+0.135}$ & $7.9_{-0.703}^{+1.697}$ & $10_{-1.17}^{+3.33}$ \\
$\rho_{\text {head1 }}(\mathrm{e} \AA-3)$ & $0.48_{-0.084}^{+0.080}$ & $0.42_{-0.003}^{+0.015}$ & $0.4_{-0.003}^{+0.002}$ \\
$\mathrm{~d}_{\text {head2 }}(\AA)$ & $7.3_{-0.669}^{+0.031}$ & $11_{-1.49}^{+0.61}$ & $9.6_{-0.432}^{+0.432}$ \\
$\rho_{\text {head2 }}(\mathrm{e} \AA-3)$ & $0.36_{-0.018}^{+0.018}$ & $0.36_{-0.004}^{+0.001}$ & $0.37_{-0.004}^{+0.003}$ \\
$\mathrm{~d}_{\text {tail }}(\AA)$ & $8.3_{-1.067}^{+0.383}$ & $8.7_{-0.698}^{+0.111}$ & $9.0_{-0.136}^{+0.136}$ \\
$\rho_{\text {tail }}(\mathrm{e} \AA-3)$ & $0.28_{-0.038}^{+0.037}$ & $0.32_{-0.019}^{+0.018}$ & $0.33_{-0.002}^{+0.003}$
\end{tabular}

The electron density of the aqueous phase was 0.336 e $\AA^{-3}$ for all samples. The error bar for each parameter was calculated based on one standard deviation from the best fit value. The electron density of the alkyl chain tail was constrained to the range $0-0.34$ e $\AA^{-3}$. The thickness of the 5FCPal head group was constrained to the minimum of $10 \AA$. 
Table S8. Structural parameters from model fitting of X-ray reflectivity curves for DOTAP and the mixtures of DOTAP and 5-FCPal $\left(\mathrm{x}_{5-\mathrm{FCPal}}=0.3,0.5,0.67\right.$ and 0.77$)$ monolayers at constant pressure of $25 \mathrm{mN} / \mathrm{m}$ at the air - liquid interface.

\begin{tabular}{|c|c|c|c|c|c|}
\hline $\mathrm{X}_{5 \text {-FCPal }}$ & o & 0.3 & 0.5 & 0.67 & 0.77 \\
\hline$\sigma_{0}(\AA)$ & $3.4_{-0.03}^{+0.062}$ & $3.3_{-0.158}^{+0.142}$ & $3.6_{-0.215}^{+0.155}$ & $3.4_{-0.066}^{+0.204}$ & $3.3_{-0.122}^{+0.158}$ \\
\hline $\mathrm{d}_{\text {head } 1}(\AA)$ & $5.2_{-2.475}^{+2.895}$ & $4.7_{-2.237}^{+4.683}$ & $5.5_{-2.521}^{+3.479}$ & $6.9_{-2.162}^{+3.938}$ & $8.9_{-2.585}^{+3.115}$ \\
\hline$\rho_{\text {head } 1}\left(\mathrm{e} \AA^{-3}\right)$ & $0.42_{-0.025}^{+0.029}$ & $0.44_{-0.02}^{+0.016}$ & $0.45_{-0.031}^{+0.018}$ & $0.45_{-0.005}^{+0.028}$ & $0.41 \stackrel{+0.041}{=0.008}$ \\
\hline & & $8.0^{-}$ & 7.0 & 8.1 & 9.7 \\
\hline$\rho_{\text {he }}$ & & $0.35_{-0.009}^{+0.006}$ & $0.35_{-0.006}^{+0.018}$ & $0.35_{-0.009}^{+0.005}$ & $0.37_{-0.011}^{+0.004}$ \\
\hline & & $11.8_{-0.64}^{+0.81}$ & 11.4 & $12.0_{-0.18}^{+0.87}$ & $10.8_{-0.6}^{+2.75}$ \\
\hline$\rho_{\text {tail }}\left(\mathrm{e} \AA^{-3}\right)$ & $0.29_{-0.009}^{+0.012}$ & $0.29_{-0.008}^{+0.005}$ & $0.31_{-0.015}^{+0.008}$ & $0.29_{-0.004}^{+0.01}$ & $0.32+$ \\
\hline
\end{tabular}

The electron density of the aqueous phase was 0.336 e $\AA^{-3}$ for all samples. The error bar for each parameter was calculated based on one standard deviation from the best fit value. The electron density of the alkyl chain tail was constrained to the range $0-0.34 \mathrm{e} \AA^{-3}$. The thickness of the head group of DOTAP was constrained to 5-6 $\AA$. The thickness of the head group in the subphase for DOTAP-5-FCPal was constrained to not exceed $20 \AA$.

Table S9. GIXD fitting parameters for DPPC and the mixtures of DPPC and 5-FCPal $\left(\mathrm{x}_{5-\mathrm{FCPal}}=\right.$ $0.3,0.5$ and 0.67 ) monolayers at constant pressure of $25 \mathrm{mN} / \mathrm{m}$ at the air - liquid interface.

\begin{tabular}{llllcc} 
& $\mathrm{Q}_{\mathrm{xy}}[1,1]$ & $\mathrm{Q}_{\mathrm{xy}}[0,2]$ & $\mathrm{Q}_{\mathrm{z}}[1,1]$ & Area $\left(\AA^{2}\right)$ & $\mathrm{t}[$ degrees $]$ \\
\hline $\mathrm{x}_{\text {5-FCPal }}=0$ & $1.33^{ \pm 0.002}$ & $1.46^{ \pm 0.001}$ & $0.73^{ \pm 0.005}$ & $48.5^{ \pm 0.2}$ & $33.30^{ \pm 0.2}$ \\
$\mathrm{x}_{\text {5-FCPal }}=0.3$ & $1.33^{ \pm 0.002}$ & $1.47^{ \pm 0.001}$ & $0.60^{ \pm 0.013}$ & $48.40^{ \pm 0.4}$ & $28.48^{ \pm 0.5}$ \\
$\mathrm{x}_{\text {5-FCPal }}=0.5$ & $1.37^{ \pm 0.002}$ & $1.47^{ \pm 0.001}$ & $0.65^{ \pm 0.014}$ & $46.63 \pm 0.3$ & $29.59^{ \pm 0.4}$ \\
$\mathrm{x}_{\text {5-FCPal }}=0.67$ & $1.38^{ \pm 0.004}$ & $1.47^{ \pm 0.002}$ & $0.60^{ \pm 0.028}$ & $46.02^{ \pm 0.8}$ & $27.068 \pm 0.9$
\end{tabular}

Area is the molecular area in the xy-plane, $\mathrm{t}$ is the tilt angle of the molecules in degree. 
Table S10. Structural parameters from model fitting of X-ray reflectivity curves for DPPC and the mixtures of DPPC and 5-FCPal ( $\mathrm{x}_{5-\mathrm{FCPal}}=0.3,0.5$ and 0.67$)$ monolayers at constant pressure of $25 \mathrm{mN} / \mathrm{m}$ at the air - liquid interface.

\begin{tabular}{|c|c|c|c|c|}
\hline $\mathbf{x}_{\text {5-FCPal }}$ & o & 0.3 & 0.5 & 0.67 \\
\hline$\sigma_{0}(\AA)$ & $3.1=0.338$ & $3.3_{-0.05}^{+0.4}$ & $3.3_{-0.086}^{+0.014}$ & $3.3_{-0.301}^{+0.3}$ \\
\hline $\mathrm{d}_{\text {head1 }}(\AA)$ & $4.1_{-1.142}^{+1.958}$ & $10.4_{-0.44}^{+0.76}$ & $11.6_{-2.02}^{+1.38}$ & $11.1_{-3.1}^{+2.5}$ \\
\hline$\rho_{\text {head } 1}\left(\mathrm{e} \AA^{-3}\right)$ & $0.47_{-0.032}^{+0.043}$ & $0.43_{-0.002}^{+0.003}$ & $0.42_{-0.007}^{+0.011}$ & $0.43_{-0.005}^{+0.005}$ \\
\hline $\mathrm{d}_{\text {head } 2}(\AA)$ & $4.5_{-2.476}^{+2.224}$ & $11.9_{-0.79}^{+2.61}$ & $8.5_{-0.496}^{+4.504}$ & $8.9_{-0.52}^{+0.08}$ \\
\hline$\rho_{\text {head2 }}\left(\mathrm{e} \AA^{-3}\right)$ & $0.41_{-0.031}^{+0.049}$ & $0.34_{-0.001}^{+0.001}$ & $0.35_{-0.007}^{+0.005}$ & $0.35_{-0.006}^{+0.002}$ \\
\hline $\mathrm{d}_{\text {taill }}(\AA)$ & $7.9_{-3.289}^{+1.011}$ & $13.4_{-0.43}^{+0.57}$ & $12.1_{-0.18}^{+0.02}$ & $12.0_{-0.45}^{+1.35}$ \\
\hline$\rho_{\text {taill }}\left(\mathrm{e} \AA^{-3}\right)$ & $0.31_{-0.009}^{+0.03}$ & $0.32_{-0.43}^{+0.005}$ & $0.31_{-0.01}^{+0.017}$ & $0.31_{-0.008}^{+0.008}$ \\
\hline $\mathrm{d}_{\text {tail2 } 2}(\AA)$ & $8.6_{-2.573}^{+1.627}$ & & & \\
\hline$\rho_{\text {tail2 }}\left(\mathrm{e} \AA^{-3}\right)$ & $0.32_{-0.016}^{+0.002}$ & & & \\
\hline
\end{tabular}

The electron density of the aqueous phase was $0.336 \mathrm{e} \AA^{-3}$ for all samples. The error bar for each parameter was calculated based on one standard deviation from the best fit value. The electron density of the alkyl chain tail was constrained to the range $0-0.34 \mathrm{e} \AA^{-3}$. The thickness of the head group of DPPC was constrained to $8 \AA$. The thickness of the head group in the subphase for DPPC-5-FCPal was constrained to not exceed 18-20 $\AA$.

Table S11. GIXD fitting parameters for DPPG and the mixtures of DPPG and 5-FCPal ( $\mathrm{x}_{5 \text {-FCPal }}$ $=0.3,0.5$ and 0.67 ) monolayers at constant pressure of $25 \mathrm{mN} / \mathrm{m}$ at the air - liquid interface.

\begin{tabular}{lllllc} 
& $\mathrm{Q}_{\mathrm{xy}}[1,1]$ & $\mathrm{Q}_{\mathrm{xy}}[0,2]$ & $\mathrm{Q}_{\mathrm{z}}[1,1]$ & Area $\left(\AA^{2}\right)$ & $\mathrm{t}[$ degrees $]$ \\
\hline $\mathrm{x}_{5-\mathrm{FCPa}}=0$ & $1.35^{ \pm 0.002}$ & $1.47^{ \pm 0.0005}$ & $0.72^{ \pm 0.005}$ & $47.38^{ \pm 0.3}$ & $32.22^{ \pm 0.2}$ \\
$\mathrm{x}_{5-\mathrm{FCPal}}=0.3$ & $1.35^{ \pm 0.002}$ & $1.47^{ \pm 0.001}$ & $0.71^{ \pm 0.01}$ & $47.68^{ \pm 0.3}$ & $32.0^{ \pm 0.4}$ \\
$\mathrm{x}_{5-\mathrm{FCPal}}=0.5$ & $1.35^{ \pm 0.003}$ & $1.47^{ \pm 0.001}$ & $0.68^{ \pm 0.01}$ & $47.46^{ \pm 0.5}$ & $30.93^{ \pm 0.4}$ \\
$\mathrm{x}_{\text {5-FCPal }}=0.67$ & $1.35^{ \pm 0.003}$ & $1.47^{ \pm 0.0005}$ & $0.70^{ \pm 0.01}$ & $47.43^{ \pm 0.4}$ & $31.51^{ \pm 0.4}$
\end{tabular}

Area is the molecular area in the xy-plane, $\mathrm{t}$ is the tilt angle of the molecules in degree. 
Table S12. Structural parameters from model fitting of X-ray reflectivity curves for DPPG and the mixtures of DPPG and 5-FCPal $\left(\mathrm{x}_{5-\mathrm{FCPal}}=0.3,0.5\right.$ and 0.67$)$ monolayers at constant pressure of $25 \mathrm{mN} / \mathrm{m}$ at the air - liquid interface.

\begin{tabular}{lcccc}
$\mathbf{x}_{\text {5-FCPal }}$ & $\mathbf{0}$ & $\mathbf{0 . 3}$ & $\mathbf{0 . 5}$ & $\mathbf{0 . 6 7}$ \\
\hline$\sigma_{0}(\AA)$ & $3.4_{-0.042}^{+0.042}$ & $3.6_{-0.088}^{0.112}$ & $3.1_{-0.11}^{+0.272}$ & $3.5_{-0.125}^{+0.095}$ \\
$\mathrm{~d}_{\text {head1 }}(\AA)$ & $4.5_{-1.091}^{+2.091}$ & $9.8_{-0.702}^{+1.902}$ & $12.1_{-1.74}^{+1.54}$ & $13.2_{-0.59}^{+0.51}$ \\
$\rho_{\text {head1 }}\left(\mathrm{e} \AA^{-3}\right)$ & $0.5_{-0.011}^{+0.022}$ & $0.46_{-0.013}^{+0.016}$ & $0.42_{-0.009}^{+0.015}$ & $0.43_{-0.004}^{+0.004}$ \\
$\mathrm{~d}_{\text {head2 } 2}(\AA)$ & $4.2_{-0.296}^{+0.2904}$ & $10.3_{-0.2}^{+0.2}$ & $9.5_{-0.5}^{+0.5}$ & $10.2_{-0.26}^{+2.26}$ \\
$\rho_{\text {head2 }}\left(\mathrm{e} \AA^{-3}\right)$ & $0.38_{-0.009}^{+0.009}$ & $0.35_{-0.005}^{+0.003}$ & $0.35_{-0.005}^{+0.004}$ & $0.35_{-0.003}^{+0.001}$ \\
$\mathrm{~d}_{\text {tail }}(\AA)$ & $17.2_{-0.06}^{+0.54}$ & $15^{-0.00} 0_{-0.1}^{+0.65}$ & $12.2_{-0.34}^{+1.06}$ & $12.4_{-0.28}^{+0.16}$ \\
$\rho_{\text {tail }}\left(\mathrm{e} \AA^{-3}\right)$ & $0.33_{-0.002}$ & $0.33_{-0.008}^{+0.003}$ & $0.32_{-0.011}^{+0.011}$ & $0.31_{-0.003}^{+0.008}$
\end{tabular}

The electron density of the aqueous phase was $0.336 \mathrm{e} \AA^{-3}$ for all samples. The error bar for each parameter was calculated based on one standard deviation from the best fit value. The electron density of the alkyl chain tail was constrained to the range $0-0.34 \mathrm{e} \AA^{-3}$. The thickness of the head group of DPPG is constrained to 8-10 $\AA$. The thickness of the head group in the subphase for DPPG-5-FCPal was constrained to not exceed 20-22 $\AA$.

Table S13.Structural parameters from model fitting of X-ray reflectivity curves for DOPC and the mixtures of DOPC and 5-FCPal $\left(\mathrm{x}_{5-\mathrm{FCPal}}=0.3,0.5\right.$, and 0.67$)$ monolayers at constant pressure of $25 \mathrm{mN} / \mathrm{m}$ at the air - liquid interface.

\begin{tabular}{|c|c|c|c|c|}
\hline $\mathbf{x}_{\text {5-FCPal }}$ & 0 & 0.3 & 0.5 & 0.67 \\
\hline$\sigma_{0}(\AA)$ & $3.4_{-0.294}^{+0.136}$ & $3.6_{-0.182}^{+0.078}$ & $3.6_{-0.063}^{+0.067}$ & $3.2_{-0.15}^{+0.29}$ \\
\hline $\mathrm{d}_{\text {head } 1}(\AA)$ & $7.3_{-2.289}^{+2.711}$ & $5.7_{-0.703}^{+0.297}$ & $6.6_{-0.615}^{+1.385}$ & $10.6_{-1.39}^{+1.01}$ \\
\hline$\rho_{\text {head } 1}\left(\mathrm{e} \AA^{-3}\right)$ & $0.43_{-0.028}^{+0.023}$ & $0.47_{-0.025}^{+0.02}$ & $0.47_{-0.024}^{+0.014}$ & $0.42_{-0.007}^{+0.036}$ \\
\hline $\mathrm{d}_{\text {head2 }}(\AA)$ & & $11.3_{-1.76}^{+0.74}$ & $11.7_{-0.85}^{+0.15}$ & $10.7_{-1.69}^{+1.61}$ \\
\hline$\rho_{\text {head} 2}\left(\mathrm{e} \AA^{-3}\right)$ & & $0.34_{-0.003}^{+0.003}$ & $0.34_{-0.002}^{+0.001}$ & $0.35_{-0.002}^{+0.005}$ \\
\hline $\mathrm{d}_{\text {tail }}(\AA)$ & $12.8_{-1.3}^{+0.7}$ & $12.1_{-0.75}^{+0.47}$ & $12.2_{-0.63}^{+0.37}$ & $10.3_{-0.33}^{+1.47}$ \\
\hline$\rho_{\text {tail }}\left(\mathrm{e} \AA^{-3}\right)$ & $0.29_{-0.015}^{+0.007}$ & $0.31_{-0.011}^{+0.004}$ & $0.31_{-0.011}^{+0.005}$ & $0.30_{-0.016}^{+0.022}$ \\
\hline
\end{tabular}

The electron density of the aqueous phase was $0.336 \mathrm{e} \AA^{-3}$ for all samples. The error bar for each parameter was calculated based on one standard deviation from the best fit value. The electron density of the alkyl chain tail was constrained to the range $0-0.34 \mathrm{e} \AA^{-3}$. The thickness of the head group of DOPC was constrained to $8 \AA$. The thickness of the head group in the subphase for DOPC-5-FCPal was constrained to not exceed 18-20 $\AA$. 

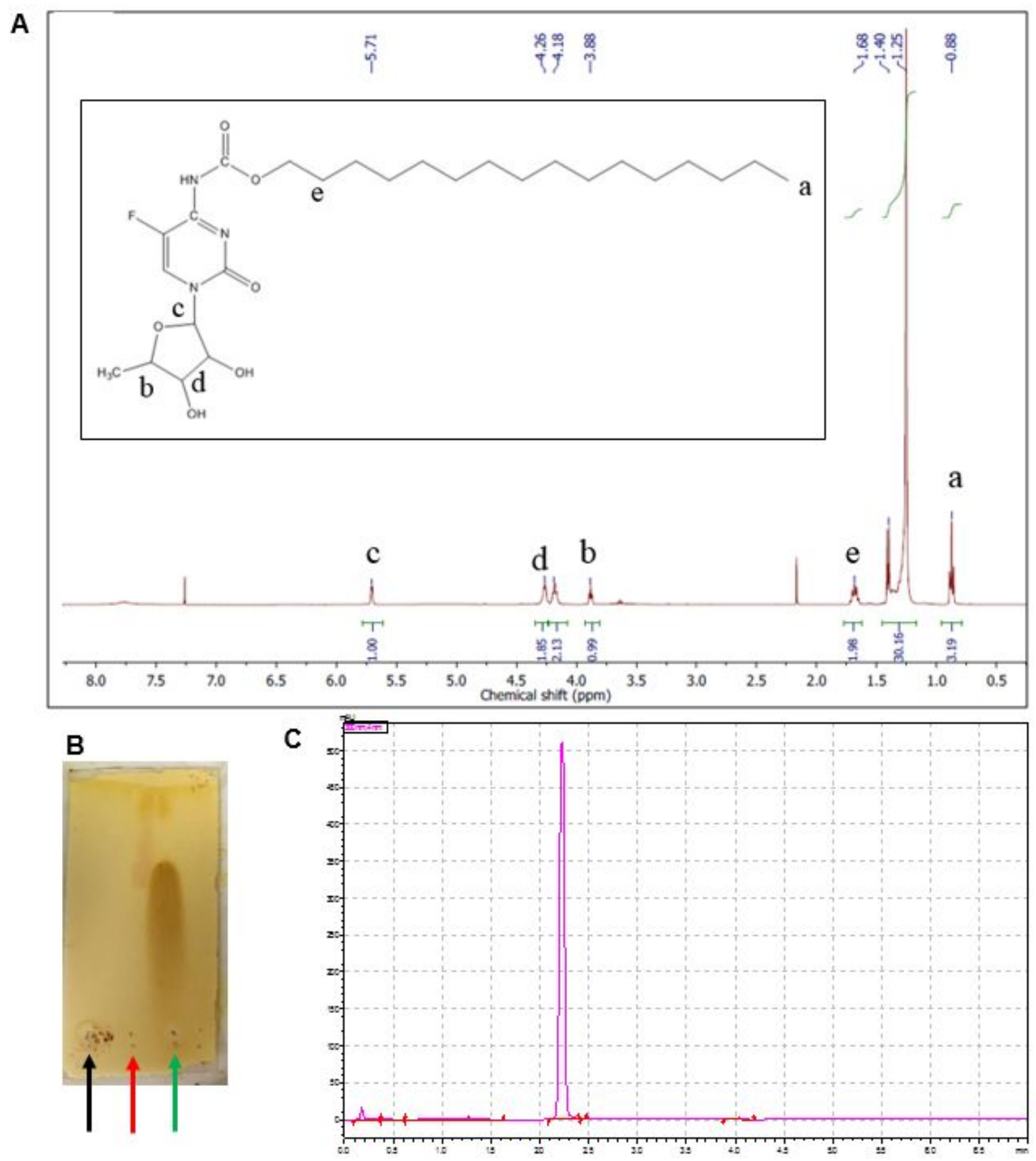

Figure S1. (A) $\mathrm{H}^{1} \mathrm{NMR}$ result confirming the structure of 5-FCPal. (B) TLC confirming the product formation during the chemical reaction. The black, red, and green arrows indicate the position of 5'DFCR, hexadecyl chloroformate, and 5-FCPal, respectively. (C) HPLC data used to confirm $99 \%$ purity of 5-FCPal. 

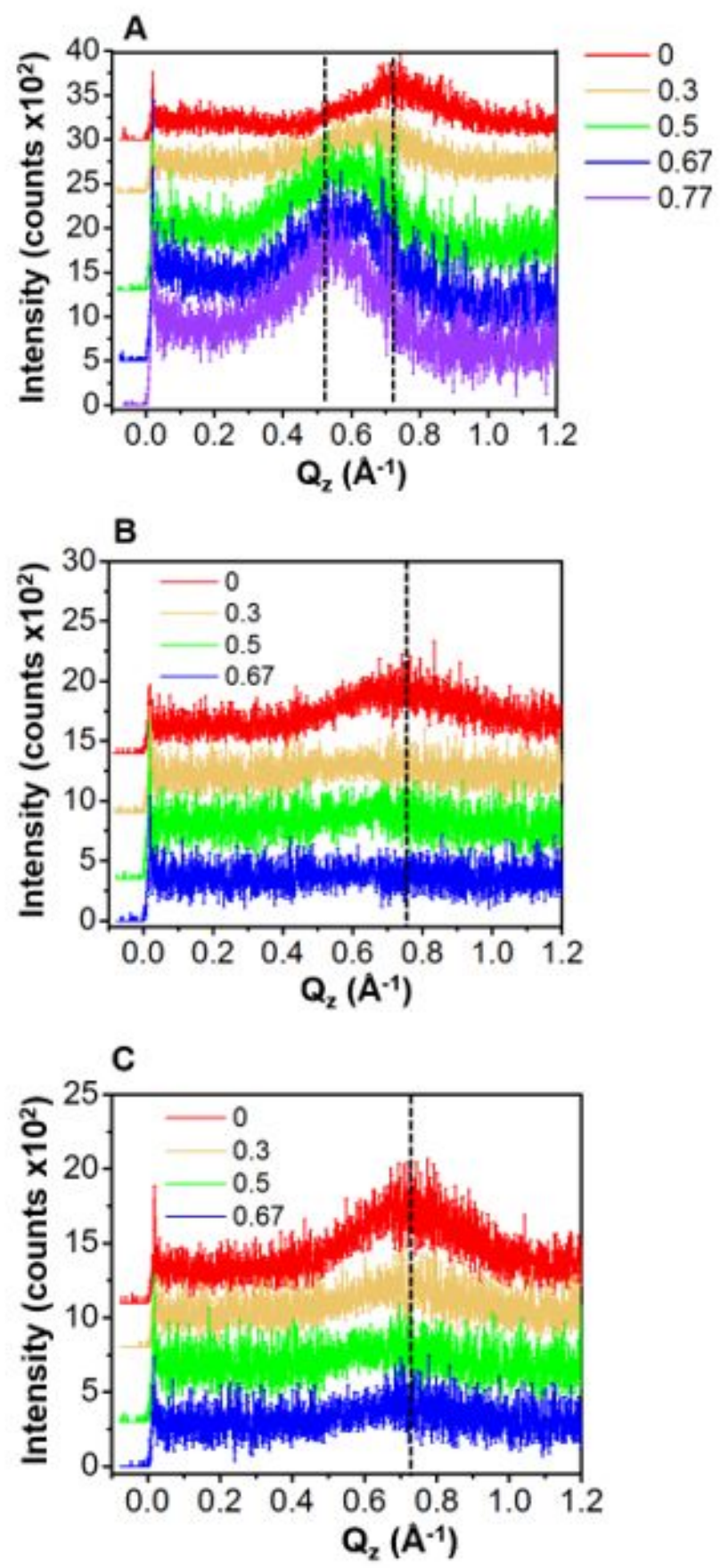

Figure S2.Bragg rods for (A) DSTAP, (B) DPPC and (C) DPPG. The positions of the Bragg rods were fitted to calculate the packing tilt angle of the lipid molecules. Bragg rods of DSTAP shifted to lower Qz as the molar fraction of 5-FCPal increased in the monolayer. This indicates the decrease in the tilt angle of DSTAP molecule as calculated in Table S3. Bragg rods of DPPC monolayer slightly shifted to lower Qz as well (Table S6). DPPG Bragg rods did not change in position. As seen in Table S9, the molecular tilt angle did not change. 


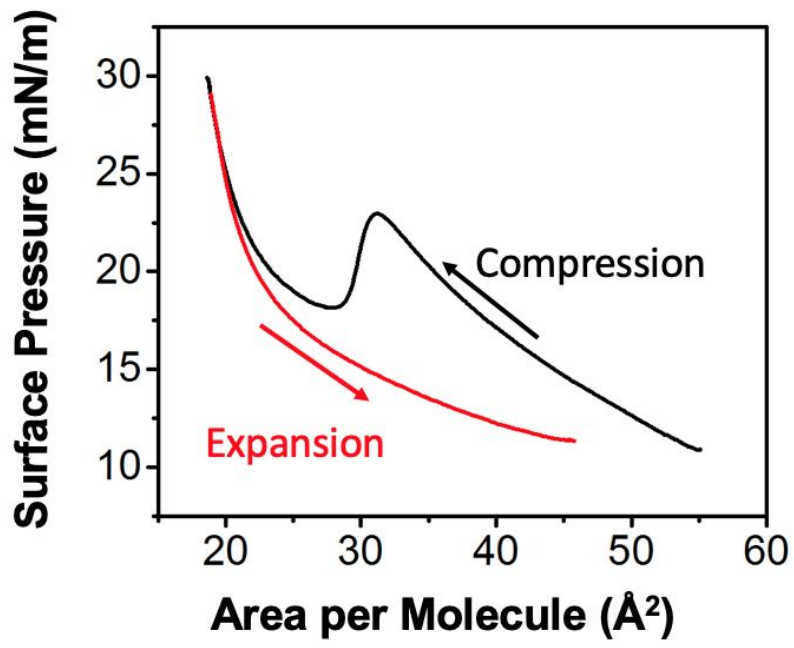

Figure S3. Compression and expansion isotherm of 5-FCPal on PBS buffer. The loop exhibits hysteresis. 

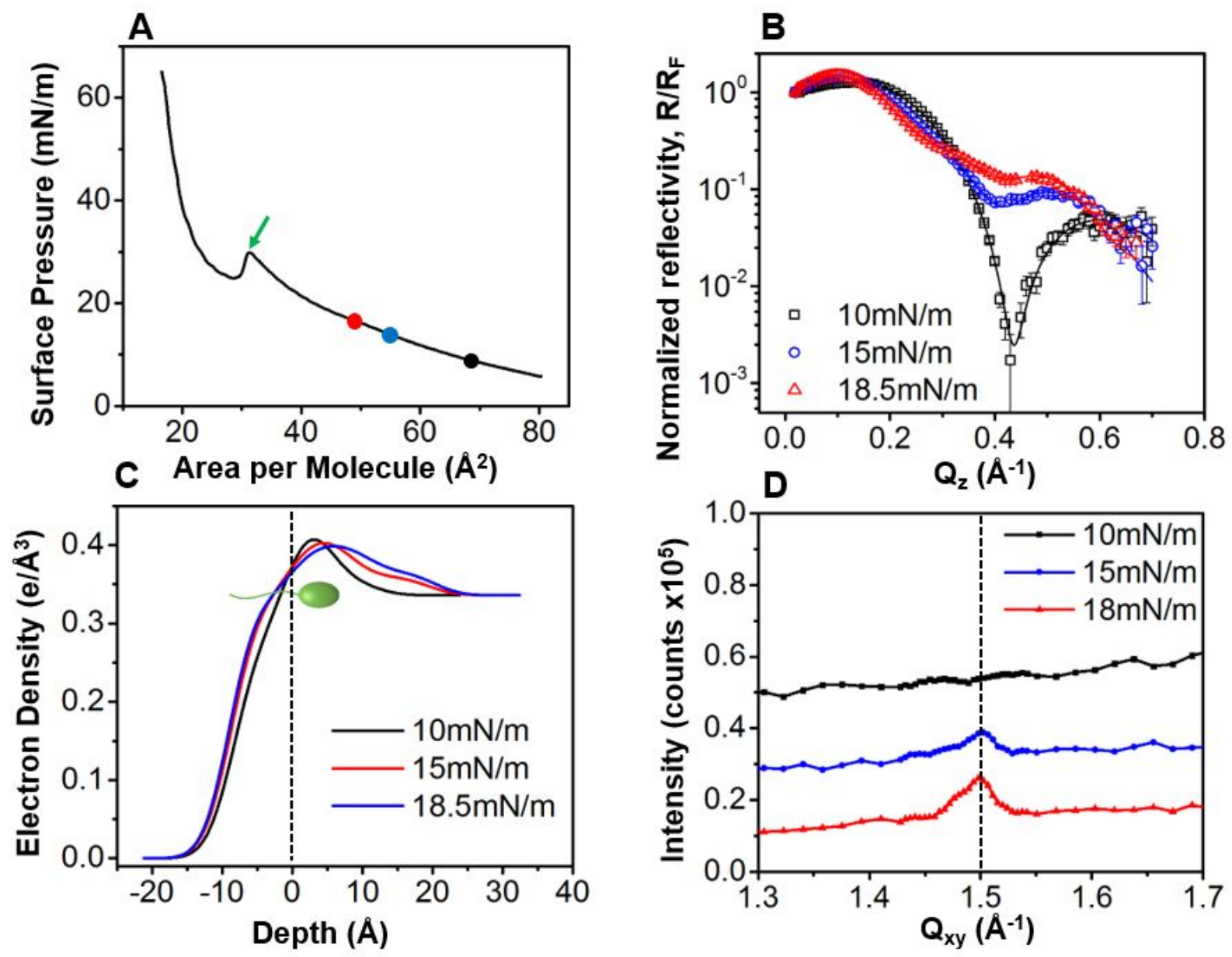

Figure S4. Organization and packing structure of pure 5-FCPal at the liquid-air interface at three different surface pressures $(10 \mathrm{mN} / \mathrm{m}, 15 \mathrm{mN} / \mathrm{m}, 18.5 \mathrm{mN} / \mathrm{m})$ before multi-layer formation. (A) Surface pressure-mean molecular area curve of pure 5-FCPal. The black, blue and red circular dot indicate the surface pressures corresponding to the X-ray measurements. The green arrow indicates the transition of 5-FCPal from monolayer to multi-layer structure. (B) Normalized reflectivity of pure $5-\mathrm{FCPal}$ monolayer at $10 \mathrm{mN} / \mathrm{m}, 15 \mathrm{mN} / \mathrm{m}$ and $18.5 \mathrm{mN} / \mathrm{m}$. The solid lines are the fittings of the XR data. The structural parameters from fitting the curves are listed in Table 1. (C) The corresponding electron density profile obtained from fitting the X-ray reflectivity. (D) Bragg peak of 5-FCPal at different surface pressures. The data is offset and the solid lines connecting the data points are provided for clarity. 


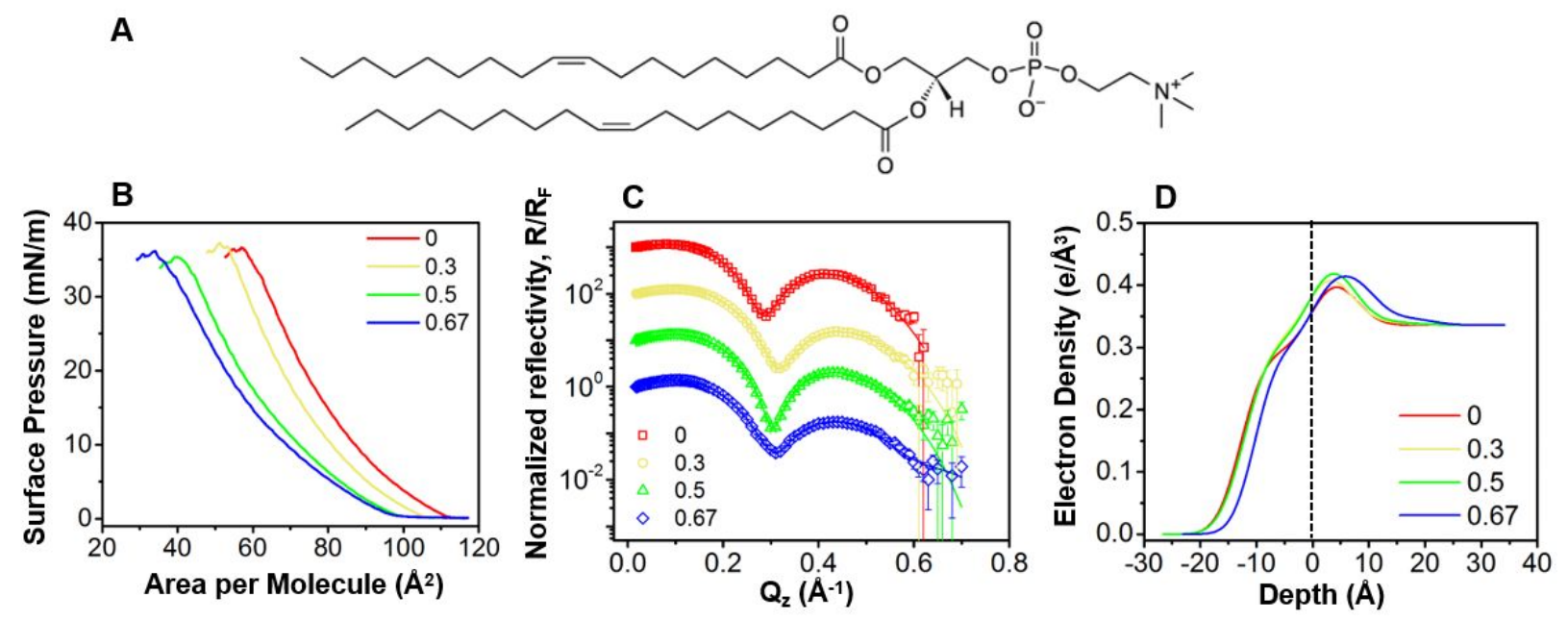

Figure S5. (A) Molecular structure of DOPC. (B) Surface pressure-mean molecular area curves of DOPC. (B) Normalized XR and electron density profiles of DOPC and 5-FCPal mixtures. (D) Electron density profiles of DOPC and 5-FCPal mixtures. The dash line $(\mathrm{z}=0)$ is artificially decided to be the water-air interface. 